\title{
FEM: mining biological meaning from cell level in Single-cell RNA sequencing data
}

\author{
Yunqing Liu ${ }^{1}$, Na Lu ${ }^{1}$, Changwei Bi ${ }^{1}$, Tingyu Han ${ }^{1}$, Guo Zhuojun ${ }^{1}$, Yunchi Zhu ${ }^{1}$, Yixin Li ${ }^{1}$, Chunpeng He ${ }^{\text {Corresp., }}{ }^{1}$, \\ Zuhong Lu ${ }^{\text {Corresp. } 1}$ \\ ${ }^{1}$ State Key Laboratory of Bioelectronics, School of Biological Science and Medical Engineering,, Southeast University, Nanjin, Jiangsu, China \\ Corresponding Authors: Chunpeng He, Zuhong Lu \\ Email address: cphe@seu.edu.cn, zhlu@seu.edu.cn
}

Background. One goal of expression data analysis is to discover the biological significance or function of genes that are differentially expressed. Gene Set Enrichment (GSE) analysis is one of the main tools for function mining that has been widely used. However, every gene expressed in a cell is valuable information for GSE for single-cell RNA sequencing (scRNA-SEQ) data and not should be discarded. Methods. We developed the functional expression matrix (FEM) algorithm to utilize the information from all expressed genes. The algorithm converts the gene expression matrix (GEM) into a FEM. The FEM algorithm can provide insight on the biological significance of a single cell. It can also integrate with GEM for downstream analysis. Results. We found that FEM performed well with cell clustering and cell-type specific function annotation in three datasets (peripheral blood mononuclear cells, human liver, and human pancreas). 
1

2 FEM: mining biological meaning from cell level in single-cell

3 RNA sequencing data

4

5

6

7

Yunqing Liu ${ }^{1}$, Na Lu${ }^{1}$, Changwei Bi ${ }^{1}$, Tingyu Han ${ }^{1}$, Zhuojun Guo ${ }^{1}$, Yunchi Zhu ${ }^{1}$, Yixin Li ${ }^{1}$, Chunpeng He${ }^{1}$, Zuhong Lu ${ }^{1}$

$8{ }^{1}$ State Key Laboratory of Bioelectronics, School of Biological Science and Medical

9 Engineering, Southeast University, Nanjing, 210096

10 Corresponding Author:

11 Chunpeng $\mathrm{He}^{1}$, Zuhong $\mathrm{Lu}^{1}$

12 Sipailou 2, Nanjing, Jiangsu Province, 210096, China

13 Email address: zhlu@seu.edu.cn

14 


\section{Abstract}

42 Background. One goal of expression data analysis is to discover the biological significance or function of genes that are differentially expressed. Gene Set Enrichment (GSE) analysis is one of the main tools for function mining that has been widely used. Every gene expressed in a cell is valuable information for GSE for single-cell RNA sequencing (scRNA-seq) data and not should be discarded.

Methods. We developed the functional expression matrix (FEM) algorithm to utilize the information from all expressed genes. The algorithm converts the gene expression matrix (GEM) into a FEM. The FEM algorithm can provide insight on the biological significance of a single cell. It can also integrate with GEM for downstream analysis.

Results. We found that FEM performed well with cell clustering and cell-type specific function annotation in three datasets (peripheral blood mononuclear cells, human liver, and human pancreas).

\section{Introduction}

RNA sequencing (RNA-seq) has been widely used as an alternative to the microarray platform over the past 10 years. It has provided valuable insight into complex biological mechanisms, ranging from cancer genomics to diverse microbial communities. Single-cell RNA-seq (scRNAseq) can facilitate new and potentially unexpected biological discoveries when compared with traditional bulk methods that profile batches of pooled cells. For example, it can provide information on complex and rare cell populations and regulatory relationships between genes and can track the trajectories of distinct cell lineages during development (Gawad, Koh \& Quake, 2016; Hwang, Lee \& Bang, 2018; Chen, Ning \& Shi, 2019).

Cells are the fundamental unit in biology. Biologists have known for centuries that multicellular organisms are characterized by a plethora of distinct cell types (Kiselev, Andrews \& Hemberg, 2019).

It is difficult to distinguish cell types from the genome sequence since all cells of an individual share the same set of genetic material. Their differences may be characterized by transcriptome similarity as defined by unsupervised clustering (Kiselev, Andrews \& Hemberg, 2019).

Bulk transcriptome studies using samples containing multiple cells cannot distinguish between the cells within the sample. Single-cell transcriptomics can address this issue (Kulkarni et al., 2019). High-throughput single-cell transcriptomics have provided unprecedented insights into the cellular diversity of tissues across diverse organisms. scRNA-seq is a promising approach in the study of the transcriptomes of organism's individual cells.

The data processing pipeline for scRNA-seq is based on bulk RNA-seq. The basic workflow includes quality control (Luecken \& Theis, 2019; Ilicic et al., 2016; Griffiths, Scialdone \& Marioni, 2018), normalization, data correction, feature selection, and dimensionality reduction, followed by cell-level and gene-level downstream analysis (Luecken \& Theis, 2019). Many genes are expressed through RNA-seq data, which is made up of pooled samples of multiple cells. However, it remains unclear whether these genes are co-expressed or just the result of 
81 mixing. Therefore, a portion of the particularly well-characterized genes must be screened for 82 downstream analysis.

83 This type of scRNA-seq data analysis has specific shortcomings. First, the characteristics of 84 single-cell data are not fully utilized. scRNA-seq data are a direct reflection of the physiological 85 state of a cell, but the aforementioned method is an analytical process based on multiple cells or 86 cell clusters. Second, it cannot effectively capture all meaningful functional groups since many 87 88 89 90 genes are filtered but may still play important roles in transient cell states. It is possible to miss meaningful biological functions since functional enrichment analysis is based on all of the cell's expressed genes, including genes that were previously filtered out. Third, the results of downstream functional analysis and upstream clustering are not integrated and visualized. To overcome these limitations, this study proposes a method based on the functional expression matrix (FEM) which converts each cell's gene expression into an enriched gene set representing a specific function.

\section{Materials \& Methods}

\section{Motivation for this approach}

Functional Gene Set Enrichment Analysis (GSEA) is often the last step of expression data analysis. A gene set usually represents a biological function, and the function will be used to refer to a gene set in subsequent analyses. Many R packages and some online sites, such as DAVID, provide enrichment analysis tools. The software takes a set of genes given by the user as input and returns functions (pathways) that are significantly enriched. Enrichment analysis methods for individual samples have also been proposed (Foroutan et al., 2018; Hänzelmann, Castelo \& Guinney, 2013).

Zhang et al. (2020) summarized the widely used GSEA algorithms. We divided their methods into three categories: methods based on dimension reduction, methods based on single samples, and methods based on phenotype or cluster.

Methods based on dimension reduction include Pagoda2 (Fan et al., 2016) and PLAGE (Tomfohr, Lu \& Kepler, 2005). These methods convert the gene expression matrix into FEM by performing dimension reduction (SVD for PLAGE and PCA for Pagoda2) on each gene set (pathway). Dimension reduction uses all samples and is not entirely based on a single cell.

Methods based on phenotype or cluster include Vision (DeTomaso et al., 2019) and z-score (Lee et al., 2008). Vision methods clusters GEM into clusters by KNN, and GSEA is based on these clusters. The Z-score method depends on phenotypes, which define each pathway activity score based on the top gene of t-test score between two conditions. These gradually join the genes in pathways to maximize the activity score and are not based on a single sample.

Methods based on the single sample include AUCell (Aibar et al., 2017), ssGSEA (Barbie et al., 2009), and GSVA (Hänzelmann, Castelo \& Guinney, 2013), which are all non-parametric test statistical methods based on gene rank. Data sparsity is the main problem when analysing data from a single cell. Therefore, the level of gene expression is not the primary consideration 
120 for a pathway, but rather, whether the gene is expressed. A method based on Fisher's exact test

121

122

123

124

125

126

127

128

129

130

131

132

133

134

135

136

137

138

139

140

141

142

143

144

145

146

147

148

149

150

151

152

153

154

155

156

157

158

or the chi-square test may have better results than the no-parameter test method based on ranking.

scRNA-seq has low capture efficiency and high dropouts due to the small amount of initial material used. scRNA-seq expression data tend to be sparse (Hicks et al., 2018b; Eraslan et al., 2019). A true zero represents the lack of expression of a gene in a specific cell due to the nontrivial distinction between true- and false-zero counts. Meanwhile, a false zero is a technical deviation. The RNA-seq technique only detects mRNA molecules that are present, therefore, a gene in the scRNA-seq dataset with a non-zero expression value indicates that at least one mRNA molecule is present. These sparse non-zero expression values provided inspiration for the possibility of performing functional GSEA analysis at the single-cell level.

Functional enrichment analysis may be performed at the single-cell transcriptome expression level given the characteristics of scRNA-seq data. The algorithm is as follows: first, replace the initial expression matrix with a FEM that allows for the direct exploration of the differences between cells from different biological perspectives. Second, the top variable function of a cell cluster obtained by a gene expression matrix (GEM) can be represented as a cell scatter plot. Third, the coverage of a functional gene set is more important than the expression of individual genes for GSEA since genes often overlap different functional groups, which makes single highexpression genes difficult to explain. For example, the activation of a signalling pathway is the result of interactions between all genes; the high expression of one gene does not mean that the pathway is activated.

The current factors affecting the discovery of cell groups are as follows: first, technical covariates must be regressed out before downstream analysis can occur since these factors introduce systematic error and confound the technical and biological variability. This error may lead to systematic differences in gene expression profiles between batches (Leek et al., 2010; Hicks et al., 2018a; Chen, Ning \& Shi, 2019). The most prominent technical covariates in singlecell data are count depth and batch. The FEM method is based on a gene set, which makes it more robust than the GEM. The measured expression levels for the genes related to a certain biological process may fluctuate between batches or single cells due to variations in sequencing processing. That is, detection for one specific high-expression gene in single cell has a certain randomness. If we can consider the expression of a set of genes related to a specific process, we can increase the detection stability for the biological process of single cells, since the number of expressed genes of a specific biological process obey a hypergeometric distribution. Second, some biological effects may affect the results of the cluster algorithms. For example, the cell cycle phase of a given cell within a cell type population may cause separation from the same cells in the clustering analysis. However, correcting for biological covariates is not always helpful in interpreting scRNA-seq data (Kolodziejczyk et al., 2015). These influencing factors often do not have uniform filtering criteria. In some cases, the cell cycle may be part of the study, or there may be a relationship between the cell cycle and other functions (Haghverdi, Buettner \& Theis, 2015; Vento-Tormo et al., 2018 ; McDavid, Finak \& Gottardo, 2016; Blasi et

Peer) reviewing PDF | (2021:04:60379:2:0:NEW 2 Nov 2021) 
159 al., 2017). The FEM method can be used to systematically survey the biological aspect of each 160 cell before downstream analysis. To this end, we developed an scRNA-seq FEM algorithm.

\section{Workflow of the proposed methods}

162 The FEM was divided into four steps (Fig. 1). Step 1: The standardized scRNA-seq GEM was

163

164

165

166

167

168

169

170

171

172

173

174

175

176

177

178

179

180

181

182

183

184

185

186

187

188

189

190

191

192

193

194

195

196

197

198

transformed into a FEM by multi-module gene enrichment analysis (gene ontology, pathway).

Step 2: The $p$-value represents the significance of enrichment, therefore, the $p$-value obtained by enrichment analysis was converted into information content (FEM). Step 3: FEM/GEM was used for data standardization, dimensionality reduction clustering, and UMAP visualization (McInnes et al., 2018). Step 4: Differentially expressed genes (DEG) and differentially expressed functions (DEF) analysis was performed based on GEM clustering (see the "GEM and FEM fusion analysis" section for details).

\section{Dataset}

Peripheral blood mononuclear cells (PBMCs) are populations of immune cells that remain in the less dense upper interface of the Ficoll layer. PBMCs include lymphocytes (T cells, B cells, and NK cells), monocytes, and dendritic cells (DCs). The frequencies of these populations vary across individuals in humans. Lymphocytes are typically in the range of 70-90\%, monocytes range from $10-30 \%$, while DCs are only present at $1-2 \%$ (NORMAN, 1995). The PBMCs dataset used in this study was downloaded from the 10X Genomics official website (https://www.10xgenomics.com/resources/datasets), which included B cells, NK cells, CD8 T cells, memory CD4 T cells, naïve CD4 T cells, DC, CD14+ monocytes, FCGR3A+ monocytes, and a small number of platelets. This dataset contained 2,700 cells in total.

The human pancreas dataset contained 2,126 cells and 10 cell types. This included alpha, ductal, endothelial, delta, acinar, beta, gamma, mesenchymal, and epsilon cells, as well as a small number of unknown cell types (Muraro et al., 2016).

The human liver dataset consisted of 777 cells, including seven types of cells: definitive endoderm cells, immediate hepatoblast cells, induced pluripotent stem cells (IPSCs), material hepatocytic cells, hepatic endoderm cells, endothelial cells, and mesenchymal stem cells (Camp et al., 2017).

\section{FEM algorithm}

\section{Selected functional groups and their profiles}

Three functional gene sets were selected from the Msigdb (v6.2) database for enrichment analysis: the Reactome pathways, gene ontology (GO), and immunologic signature gene sets (Liberzon et al., 2011) (Table 1).

\section{Validation gene set}

ImmuneSigDB (Godec et al., 2016) contained a gene set composed of differentially expressed genes from human and mouse immune-related cells. These were collected from the literature with corresponding expression data in the Gene Expression Omnibus (GEO) database. All data were manually reviewed and standardized using the same method. The differentially expressed FDR value was less than 0.02 , and the genes were sorted using the mutual information algorithm. No more than 300 genes were selected for each gene set. 
199

200

201

202

203

204

205

206

207

208

209

210

211

212

213

214

215

216

217

218

219

220

221

222

223

224

225

226

227

228

229

230

231

232

\section{Gene-functional group conversion}

The cells' non-zero-expressed genes (i.e., genes in which the read count or normalized expression values, such as RPKM and FPKM, were not zero) in the GEM were extracted first. In the second step, an enrichment analysis score for each cell was calculated. The enrichment analysis method was based on Fisher's exact test using the Python SciPy package. The Fisher exact test is a statistical test based on a hypergeometric distribution that is used to determine if there are non-random associations between two categorical variables, or to test whether the theoretical value is consistent with the actual value.

$$
P=\frac{\left(\begin{array}{l}
K \\
k
\end{array}\right)\left(\begin{array}{l}
N-K \\
n-k
\end{array}\right)}{\left(\begin{array}{l}
N \\
n
\end{array}\right)}
$$

Here, $N$ represents the total number of background genes, which is defined as all nonredundant genes in all gene sets taken from a species' function database, such as Reactome pathway or GO database. $K$ represents the number of genes in a particular gene set, $n$ represents the number of non-zero genes in a single cell, and $k$ represents the number of genes present in both $K$ and $n$. The Bonferroni correction was used to counteract the problem of multiple comparisons, but this is optional.

\section{Expression value conversion based on information content}

Information content measures the average rate of information from data. The smaller the $p$-value, the greater the amount of information. For the adjusted $p$-value of enrichment of a gene set, the null hypothesis is that there is no significant enrichment. So, the smaller the adjusted $p$-value, the more significant the enrichment of the gene set (rather than stochastic). Therefore, here the information content was used as a measure of the level of expression of a functional group.

$$
G S_{i, j}=-\log \left(a d j-p_{i, j}\right)
$$

Here, $i$ is the $i t h$ gene set, $j$ is the $j$ th cell, and $a d j-p_{i, j}$ represents the adjusted $p$-value in the $i t h$ gene set in the $j$ th cell.

\section{Algorithm optimization}

Fisher's exact test is a time-consuming process. For single-cell data, a statistical test would be required for each function of each cell. Therefore, when the number of cells is large, the computation time would be untenable. Therefore, the algorithm was optimized with the addition of multi-core computing.

Optimization of the algorithm can be illustrated using the symbols in section "Gene-functional group conversion". First, because $N$ and $K$ were invariable for all cells, these values were stored in memory to avoid recalculation with each replicate. Second, the gene expression matrix was transformed into 0,1 matrix $A$, where 1 represents the expression of gene $i$ in cell $j$ and 0 represents the non-expression of gene $i$ in cell $j$. The gene set was also transformed into 0,1 
233 matrix $B$, where 1 represents the presence of gene $i$ in set $s$ and 0 represents the absence of gene $234 i$ in set $s$. The element $(k)$ in the product $A \times B^{T}$ of the two matrices represents the number of 235 genes expressed by cell $j$ in set $s$ (Fig. 2).

236 Cluster and differentially expressed gene detection based on FEM and 237 integration of GEM and FEM by Seurat

238 Analysis tools for scRNA-seq data

239 A number of integrated data analysis software packages and platforms exist, including Seurat 240 (Butler et al., 2018), Scater (McCarthy et al., 2017), and Scanpy (Wolf, Angerer \& Theis, 2018).

241 Seurat provides integrated environments (including sample and feature selection, data

242 standardization, dimensionality reduction, clustering, and visualization) to explore massive

243 scRNA-seq datasets (Luecken \& Theis, 2019). Seurat was used in this study for normalization, 244 dimensionality reduction, clustering, and visualization.

245 Dimensionality reduction

246 The dimensions of single-cell expression matrices were further reduced after feature selection by 247 dedicated dimensionality-reduction algorithms. These algorithms, such as principal component

248

249

250

251

252

253

254

255

256

257

258

259

260

261

262

263

264

265

266

267

268

269

270

271

272 analysis (PCA), embed the expression matrix into a low-dimensional space, which is designed to capture the underlying structure in the data in as few dimensions as possible (Luecken \& Theis, 2019; Eraslan et al., 2019). Our data were converted into a linear combination of the first $N$ principal components by the PCA algorithm. The value corresponding to the 'elbow' point was taken as the value of $N$.

\section{Clustering}

Single cells were clustered during the analysis of scRNA-seq transcriptome profiles. This may reveal cell subtypes and infer cell lineages based on the relationships among cells. Several software packages support the cluster analysis of scRNA-seq data (Petegrosso, Li \& Kuang, 2019). Seurat was used for clustering based on a graphical approach. The cluster function was set to $0.4-1.2$ according to the data. All parameters are show in Table S5

\section{Differential expression between clusters}

Differential expression analysis is helpful for finding the significant DEG/DEF between distinct subpopulations or groups of cells (Petegrosso, Li \& Kuang, 2019). Seurat was used to determine the subsets of functions that exhibited a high variation between clusters.

\section{GEM and FEM fusion analysis}

FEM is limited in that it only considers the presence or absence of gene expression without considering the expression value of the gene. Therefore, FEM cannot replace GEM-based methods in cell classification and type identification. In the present study, data from the GEM and FEM were used for fusion analysis (Fig. 1). The multi-modal data analysis module of Seurat was used for polymerization analysis to combine the GEM and FEM results (Stuart et al., 2019; Stuart \& Satija, 2019). GEM was used for clustering because it contains information about gene expression values and can better distinguish the differences between cells. FEM reduces all of the genes of the same function to one dimension. If two cells express different genes of the same function, they will be clustered together in FEM, but they may be separated in GEM since they 
273 are different genes. We also tested the Differential Expression Functions between Clusters based

274 on GEM (GC-DEF) and the Differentially Expressed Functions between Clusters based on FEM 275 (FC-DEF).

276 The feature number selection, scaling ratio, PCA, and clustering parameter selection were 277 appropriately adjusted according to circumstances (Table S5), following the Seurat instructions.

278 In brief, we use the VlnPlot visualization function in the Seurat package to select the feature 279 number and scaling ratio value for subsequent analysis. The feature number and the scaling ratio 280 are respectively taken as the maximum value of ordinate that can include all the points after

281

282

283

284

285

286

287

288

289

290

291

292

293

294

295

296

297

298

299

300

301

302

303

304

305

306

307

308 excluding individual excessively high outliers. We can select the number of principal components by observing the corresponding abscissa value at the "elbow" point of the Elbow plot by Seurat. If the number of cell types is known, the clustering parameters were set so that the number of clusters was greater than or equal to the number of actual cell types. If the number of cell types is unknown, we can adjust the number of clusters by setting the resolution parameter of FindClusters function in Seurat. Like most clustering algorithms, there is no uniform standard to define a specific number of clusters. Users can try multiple parameters to get a more appropriate number of clusters. All parameters in this paper are detailed in Table S5.

\section{Evaluation of FEM clustering results}

We defined an evaluation score, $S C$, to compare the overlap between clusters based on FEM clustering and real cell types. The number of clusters was related to specific parameters, therefore, each cluster should contain only one cell type as much as possible. Additionally, the cluster's number should be greater than or equal to the number of cell types. Therefore, the clustering parameters were set so that the number of clusters was greater than or equal to the number of actual cell types. For each cluster $i, S C_{i}$ was measured by the consistency of the cell type within the cluster (the proportion of the cell type with the largest number of cells to the total number of cells in the cluster). We used the following formula to evaluate clustering results:

$$
S C_{i}=\frac{c_{i, \max }}{c_{i, T}}
$$

$c_{i, \max }$ represents the cell type with the largest number of cells in the $i$-th cluster. $c_{i, T}$ represents the total number of all cells in the $i$-th cluster.

\section{Data availability}

Publicly available scRNA-seq datasets were used in this study. The PBMCs dataset was downloaded from the 10X Genomics dataset page (https://cf.10xgenomics.com/samples/cell/pbmc3k/pbmc3k_filtered_gene bc matrices.tar.gz). The liver and pancreas scRNA-seq data can be accessed in the NCBI Gene Expression Omnibus (GEO) under accession numbers: GSE81252 (https://www.ncbi.nlm.nih.gov/geo/query/acc.cgi?acc=GSE81252), GSE85241 (https://www.ncbi.nlm.nih.gov/geo/query/acc.cgi?acc=GSE85241).

\section{Code availability}


309 The FEM software and related scripts have been deposited in the GitHub repository

310 (https://github.com/qingyunpkdd/single cell fem).

311

312 Results

313 FEM can separate different cell types

314 The Reactome pathway gene sets have only 5,741 unique genes and a large amount of gene

315 information was lost when using the FEM algorithm. In comparison GO gene sets had 15,578

316 unique genes. Therefore, GO gene set-based FEM was used for cluster analysis. The Reactome

317 pathway gene sets were used in the analysis of GC-DEF and their cluster results are shown in

318 Figure S1. GO-based FEM results are shown in Figures 3, 4, and 5.

319 Three data sets (PBMCs, liver, pancreas) were used to replace GEM for dimensionality

320 reduction and clustering to verify whether the GO-based FEM algorithm could separate different

321 cell types. The number of clusters was artificially adjusted and the number of clusters was set to

322 be greater than or equal to the number of actual cell types. This was done so that a cluster

323 contained only one main cell type, which allowed us to better distinguish the different cell types.

324 We needed to discover different cell groups and determine the relationship between different

325 cell types for single-cell scRNA-seq data. These cells may have similar functions or a common

326 recent differentiation origin. The FEM method may help determine functionally similar cells.

327 Cells were noted to be different subtypes of the same cell if the two groups of cells were far apart

328 in GEM, but close or partially overlapped in FEM. This result may also indicate that the two

329 groups of cells may perform similar functions.

330 We performed GEM clustering and GO-based FEM clustering on the scRNA-seq data, and

331 compared the two clusters. The FEM clustering results were also compared with real cell types to

332 determine the similarity of cell functions. A low $S C$ score in a particular cluster $i$ indicated that

333 the cluster may contain multiple cell types. A moderate number of a certain cell type $A$ in the

334 cluster $i$ was defined as the main cell type $M C_{i}$. For a cluster, all $M C s$ may have the same or

335 similar functions.

336 The results show that the FEM method can distinguish different types of cells that have

337 different functions on three data sets $($ Fig. 3, 4, 5). Figures 3, 4, and 5 show that FEM can find

338 cells with similar origins or different subgroups of a cell. Figure 3 shows three clusters (G1, G2,

339 G3) that have a low $S C$ score and all of the T cells overlapped in cluster G3. The $M C_{i}$ of NK

340 cells and $\mathrm{T}$ cells in cluster $\mathrm{G} 2$ literature show that they have a common differentiation origin

341 T/NK cell progenitor. G1's $M C_{i}$ contained only monocytes (Lim et al., 2013). The FEM in

342 Figure 4 helped determine the relationship of definitive endoderm cells and hepatic endoderm

343 cells (Camp et al., 2017). Figure 5 illustrates that alpha, beta, gamma, epsilon, and delta cells

344 form one cluster in the FEM clusters, and that the cell differentiation tree verifies that they have

345 a common endocrine progenitor (Jacobson \& Tzanakakis, 2017).

346 FC-DEF can directly detect the functional differences between clusters 
347 GC-DEF can reveal the expression of specific functions from the UMAP(t-SNE) diagram of GEM and 348 the results of GC-DEF may aid GEM in determining the functions of a specific group. Official

349

350

351

352

353

354

355

356

357

358

359

360

361

362

363

364

365

366

367

368

369

370

371

372

373

374

375

376

377

378

379

380

381

382

383

384

385

386 data pre-processing in the PBMC dataset included the removal of cells with excessive mitochondrial genes ( $>5 \%$, quality control) and those with too many $(>2,500)$ or too few $(<$ 200) features (Bittersohl \& Steimer, 2016). Some cells may have been filtered out in the data pre-processing stage whereas our method did not filter out any cells. Indeed, the filtered cells were found to be located above the NC cells. Using the GC-DEF method, this cell group was found to have a highly expressed cell proliferation-related pathway. Thus, these cells were identified as proliferative (Fig. 6).

The most significantly expressed pathway, "hemostasis", was located in the platelet clusters. The "innate immune system" pathway was significantly expressed in the monocyte, DC cell, NK cell, and platelet cell populations, which was consistent with literature results (NORMAN, 1995). The top five highly expressed functions were consistent with the cell type characteristics. The other FC-DEF and GC-DEF results are provided in Table S1.

Table 2 shows that most of the corresponding cells were closely related to their corresponding top functions, such as the high expression of "Reactome regulation of beta cell development" in beta cells and the high expression of "Reactome gluconeogenesis" in mature stem cells. All other results are shown in Table s1.

GO-based GC-DEF results was consistent with the pathway-based methods and literature results (NORMAN, 1995). All other results are presented in Table 3 and Table S1, S2, S3 and S4.

\section{Difference between GO enrichment analysis results based on DEG and FEM}

The general GSE analysis was based on DEGs between groups (cell types or clusters). The FEM-based gene set (function) enrichment can be defined as the DEF between groups. The results (Table S5, p-value threshold $\leq 0.05$ ) show that: 1 . the enrichment analysis results obtained by the two methods are quite different. 2. There are more GO items based on the FEM method than the method based on DEGs.

The possible explanation for the first point is that the two methods are based on different data, and the FEM method is an enrichment result of a single cell. The DEG method is based on results from cell clusters. FEM uses all expressed genes in a cell to calculate GO enrichment, while the DEG method only uses those differentially expressed genes, which may explain why there are more GO items based on the FEM method than the DEGs method. The FEM method focuses on the "differentially expressed GO items", and the DEG method focuses on "the enriched GO items of differentially expressed genes".

\section{Validation with an immune dataset}

The Immunologic Signatures Collection (ImmuneSigDB) (Liberzon et al., 2011) was used as a validation dataset to test whether the proposed method could detect sets of genes that had been identified as up-regulated or down-regulated by traditional methods. The ImmuneSigDB is composed of gene sets that represent cell types, states, and perturbations within the immune system (Godec et al., 2016). Figure 7 shows that within the bulk RNA-seq dataset, the cell types 
387

388

389

390

391

392

393

394

395

396

397

398

399

400

401

402

403

404

405

406

407

408

409

410

411

412

413

414

415

416

417

418

419

420

421

422

423

424

425

from the up-regulated expression marker gene set were highly expressed using the method proposed in this study. This demonstrated the efficacy of the proposed method for detecting celltype-specific gene sets.

\section{Discussion}

We showed that FEM can be used for cell clustering. It also can merge the GEM method for downstream differential expression analysis to find cell type-specific functions.

It was necessary to evaluate the impact of some biological effects on the cell clusters, such as the impact of cell cycles on cell-type clustering results. Within the same cell type, cells in the cell proliferation cycle and non-proliferating cells may be significantly separated in the cluster and UMAP plots, which affects the final cell type identification and differential expression analysis. In some studies, these cells are removed, while they may still be of interest and there is currently no uniform standard for handling these cells. The method proposed in this study directly converts the expression of genes in cells to the expressions of functions and cells can be screened according to their FEM score at any stage of processing.

It should be noted that FEM only considers the presence and absence of gene expression, without considering the influences of gene-expression values. Therefore, the proposed method and gene-expression-based methods are complementary and should not be considered as alternatives to one another.

We have proven that the FEM-based method can also be applied to cell clustering, and verified the accuracy of cell clustering when compared with real cell types. A direct analysis of the differential expression function between the groups and visually elucidating the differences in specific functions helps to intuitively understand the functions of cells. FEM and GEM were used as two different data sources, while multi-omics data fusion analysis was reused in Seurat and other tools to show the functional results of GEM analysis.

The information that each cell can provide may be valuable for single-cell transcriptome data. The same cell type may also be in different physiological states (for example: different stages of the cell cycle, different metabolic states). Cells in a differentiation stage may have a series of cell states. Therefore, it is necessary to develop data analysis tools for individual cells to find those few but possibly important cells, such as those cells that are in a transient state of differentiation.

\section{Conclusions}

The final step in the analysis of gene expression data is to interpret the biological significance of the genes based on a hypergeometric test between the listed genes of interest and the set of genes representing specific functions. If each of the first $n$ genes obtained by differential expression analysis represents a function that does not overlap with the other, then the enrichment analysis will fail. If most genes of a function are expressed in a cell and are screened out in the process of gene selection, this function will also be missed. GSEA at the single-cell level effectively avoids these problems based on the characteristics of single-cell data. The results of the present study 
426

427

428

429

430

431

432

433

434

435

436

437

438

439

440

441

442

443

444

445

446

447

448

449

450

451

452

453

454

455

456

457

458

459

460

461

462

463

464

465

466

467

showed that direct enrichment analysis at the single-cell level is feasible and yields powerful results.

We preliminarily discussed the applicability of the FEM method and the FEM have a wider range of practical applications. The possible applications of the FEM method include: determining the functional differences between different groups; analysing the function of a few outlier cells; analysing the state of a small number of cells on the cell trajectory in the pseudotrajectory analysis; analysing which cell groups are more similar in function by comparing GEM and FEM clustering; determining marker quality for different cell groups compared with genes (though there is a need for future validation); and more differential functions were found compared with the GEM method. The disadvantages of the FEM method are: the influence of gene expression is not considered; it is impossible to distinguish cell groups with similar functions; and the function of differential expressions and the result of DEG-based enrichment are quite different, and they cannot be substituted for each other.

\section{Acknowledgements}

We would like to thank the Southeast University State Key Laboratory of Bioelectronics for providing the computing resources used in our research.

\section{References}

Aibar S, González-Blas CB, Moerman T, Huynh-Thu VA, Imrichova H, Hulselmans G, Rambow F, Marine JC, Geurts P, Aerts J, Van Den Oord J, Atak ZK, Wouters J, Aerts S. 2017. SCENIC: Single-cell regulatory network inference and clustering. Nature Methods 14. DOI: $10.1038 /$ nmeth.4463.

Barbie DA, Tamayo P, Boehm JS, Kim SY, Moody SE, Dunn IF, Schinzel AC, Sandy P, Meylan E, Scholl C, Fröhling S, Chan EM, Sos ML, Michel K, Mermel C, Silver SJ, Weir BA, Reiling JH, Sheng Q, Gupta PB, Wadlow RC, Le H, Hoersch S, Wittner BS, Ramaswamy S, Livingston DM, Sabatini DM, Meyerson M, Thomas RK, Lander ES, Mesirov JP, Root DE, Gilliland DG, Jacks T, Hahn WC. 2009. Systematic RNA interference reveals that oncogenic KRAS-driven cancers require TBK1. Nature 462. DOI: 10.1038/nature08460.

Bittersohl H, Steimer W. 2016. Intracellular Concentrations of Immunosuppressants. In: Personalized Immunosuppression in Transplantation: Role of Biomarker Monitoring and Therapeutic Drug Monitoring. Elsevier Inc., 199-226. DOI: 10.1016/B978-0-12-8008850.00009-6.

Blasi T, Buettner F, Strasser MK, Marr C, Theis FJ. 2017. CgCorrect: A method to correct for confounding cell-cell variation due to cell growth in single-cell transcriptomics. Physical Biology. DOI: 10.1088/1478-3975/aa609a.

Butler A, Hoffman P, Smibert P, Papalexi E, Satija R. 2018. Integrating single-cell transcriptomic data across different conditions, technologies, and species. Nature Biotechnology. DOI: 10.1038/nbt.4096.

Camp JG, Sekine K, Gerber T, Loeffler-Wirth H, Binder H, Gac M, Kanton S, Kageyama J, Damm G, Seehofer D, Belicova L, Bickle M, Barsacchi R, Okuda R, Yoshizawa E, Kimura M, Ayabe H, Taniguchi H, Takebe T, Treutlein B. 2017. Multilineage communication 
468

469

470

471

472

473

474

475

476

477

478

479

480

481

482

483

484

485

486

487

488

489

490

491

492

493

494

495

496

497

498

499

500

501

502

503

504

505

506

507

508

509

510

511

512

513

regulates human liver bud development from pluripotency. Nature 546. DOI:

10.1038 /nature22796.

Chen G, Ning B, Shi T. 2019. Single-cell RNA-seq technologies and related computational data analysis. Frontiers in Genetics 10:1-13. DOI: 10.3389/fgene.2019.00317.

DeTomaso D, Jones MG, Subramaniam M, Ashuach T, Ye CJ, Yosef N. 2019. Functional interpretation of single cell similarity maps. Nature Communications 10. DOI: 10.1038/s41467-019-12235-0.

Eraslan G, Simon LM, Mircea M, Mueller NS, Theis FJ. 2019. Single-cell RNA-seq denoising using a deep count autoencoder. Nature Communications 10:1-14. DOI: 10.1038/s41467018-07931-2.

Fan J, Salathia N, Liu R, Kaeser GE, Yung YC, Herman JL, Kaper F, Fan JB, Zhang K, Chun J, Kharchenko P V. 2016. Characterizing transcriptional heterogeneity through pathway and gene set overdispersion analysis. Nature Methods 13. DOI: 10.1038/nmeth.3734.

Foroutan M, Bhuva DD, Lyu R, Horan K, Cursons J, Davis MJ. 2018. Single sample scoring of molecular phenotypes. BMC Bioinformatics 19:1-10. DOI: 10.1186/s12859-018-2435-4.

Gawad C, Koh W, Quake SR. 2016. Single-cell genome sequencing: Current state of the science. Nature Reviews Genetics 17:175-188. DOI: 10.1038/nrg.2015.16.

Godec J, Tan Y, Liberzon A, Tamayo P, Bhattacharya S, Butte AJ, Mesirov JP, Haining WN. 2016. Compendium of Immune Signatures Identifies Conserved and Species-Specific Biology in Response to Inflammation. Immunity 44:194-206. DOI: 10.1016/j.immuni.2015.12.006.

Griffiths JA, Scialdone A, Marioni JC. 2018. Using single - cell genomics to understand developmental processes and cell fate decisions. Molecular Systems Biology. DOI: $10.15252 / \mathrm{msb} .20178046$.

Hänzelmann S, Castelo R, Guinney J. 2013. GSVA: Gene set variation analysis for microarray and RNA-Seq data. BMC Bioinformatics 14:1-21. DOI: 10.1186/1471-2105-14-7.

Haghverdi L, Buettner F, Theis FJ. 2015. Diffusion maps for high-dimensional single-cell analysis of differentiation data. Bioinformatics. DOI: 10.1093/bioinformatics/btv325.

Hänzelmann S, Castelo R, Guinney J. 2013. GSVA: Gene set variation analysis for microarray and RNA-Seq data. BMC Bioinformatics 14:1-21. DOI: 10.1186/1471-2105-14-7.

Hicks SC, Townes FW, Teng M, Irizarry RA. 2018a. Missing data and technical variability in single-cell RNA-sequencing experiments. Biostatistics. DOI: 10.1093/biostatistics/kxx053.

Hicks SC, Townes FW, Teng M, Irizarry RA. 2018b. Missing data and technical variability in single-cell RNA-sequencing experiments. Biostatistics 19:562-578. DOI: 10.1093/biostatistics/kxx053.

Hwang B, Lee JH, Bang D. 2018. Single-cell RNA sequencing technologies and bioinformatics pipelines. Experimental and Molecular Medicine 50. DOI: 10.1038/s12276-018-0071-8.

Ilicic T, Kim JK, Kolodziejczyk AA, Bagger FO, McCarthy DJ, Marioni JC, Teichmann SA. 2016. Classification of low quality cells from single-cell RNA-seq data. Genome Biology. DOI: 10.1186/s13059-016-0888-1.

Jacobson EF, Tzanakakis ES. 2017. Human pluripotent stem cell differentiation to functional pancreatic cells for diabetes therapies: Innovations, challenges and future directions. Journal of Biological Engineering 11. DOI: 10.1186/s13036-017-0066-3.

Kiselev VY, Andrews TS, Hemberg M. 2019. Challenges in unsupervised clustering of singlecell RNA-seq data. Nature Reviews Genetics 20:273-282. DOI: 10.1038/s41576-018-00889 .

PeerJ reviewing PDF | (2021:04:60379:2:0:NEW 2 Nov 2021) 
514 Kolodziejczyk AA, Kim JK, Svensson V, Marioni JC, Teichmann SA. 2015. The Technology

515

516

517

518

519

520

521

522

523

524

525

526

527

528

529

530

531

532

533

534

535

536

537

538

539

540

541

542

543

544

545

546

547

548

549

550

551

552

553

554

555

556

557

558 and Biology of Single-Cell RNA Sequencing. Molecular Cell. DOI: 10.1016/j.molcel.2015.04.005.

Kulkarni A, Anderson AG, Merullo DP, Konopka G. 2019. Beyond bulk: a review of single cell transcriptomics methodologies and applications. Current Opinion in Biotechnology 58:129136. DOI: 10.1016/j.copbio.2019.03.001.

Leek JT, Scharpf RB, Bravo HC, Simcha D, Langmead B, Johnson WE, Geman D, Baggerly K, Irizarry RA. 2010. Tackling the widespread and critical impact of batch effects in highthroughput data. Nature Reviews Genetics. DOI: 10.1038/nrg2825.

Lee E, Chuang HY, Kim JW, Ideker T, Lee D. 2008. Inferring pathway activity toward precise disease classification. PLoS Computational Biology 4. DOI: 10.1371/journal.pcbi.1000217.

Liberzon A, Subramanian A, Pinchback R, Thorvaldsdóttir H, Tamayo P, Mesirov JP. 2011. Molecular signatures database (MSigDB) 3.0. Bioinformatics 27:1739-1740. DOI: 10.1093/bioinformatics/btr260.

Lim WF, Inoue-Yokoo T, Tan KS, Lai MI, Sugiyama D. 2013. Hematopoietic cell differentiation from embryonic and induced pluripotent stem cells. Stem Cell Research and Therapy 4. DOI: $10.1186 /$ scrt222.

Luecken MD, Theis FJ. 2019. Current best practices in single - cell RNA - seq analysis: a tutorial. Molecular Systems Biology 15:e8746. DOI: 10.15252/msb.20188746.

McCarthy DJ, Campbell KR, Lun ATL, Wills QF. 2017. Scater: Pre-processing, quality control, normalization and visualization of single-cell RNA-seq data in R. Bioinformatics. DOI: 10.1093/bioinformatics/btw777.

McDavid A, Finak G, Gottardo R. 2016. The contribution of cell cycle to heterogeneity in single-cell RNA-seq data. Nature Biotechnology. DOI: 10.1038/nbt.3498.

McInnes L, Healy J, Saul N, Großberger L. 2018. UMAP: Uniform Manifold Approximation and Projection. Journal of Open Source Software 3. DOI: 10.21105/joss.00861.

Muraro MJ, Dharmadhikari G, Grün D, Groen N, Dielen T, Jansen E, van Gurp L, Engelse MA, Carlotti F, de Koning EJP, van Oudenaarden A. 2016. A Single-Cell Transcriptome Atlas of the Human Pancreas. Cell Systems 3. DOI: 10.1016/j.cels.2016.09.002.

NORMAN P. 1995. Immunobiology: The immune system in health and disease. Journal of Allergy and Clinical Immunology. DOI: 10.1016/s0091-6749(95)70025-0.

Petegrosso R, Li Z, Kuang R. 2019. Machine learning and statistical methods for clustering single-cell RNA-sequencing data. Briefings in Bioinformatics 00:1-15. DOI: 10.1093/bib/bbz063.

Stuart T, Butler A, Hoffman P, Hafemeister C, Papalexi E, Mauck WM, Hao Y, Stoeckius M, Smibert P, Satija R. 2019. Comprehensive Integration of Single-Cell Data. Cell 177:18881902.e21. DOI: 10.1016/j.cell.2019.05.031.

Stuart T, Satija R. 2019. Integrative single-cell analysis. Nature Reviews Genetics 20:257-272. DOI: $10.1038 / \mathrm{s} 41576-019-0093-7$.

Tomfohr J, Lu J, Kepler TB. 2005. Pathway level analysis of gene expression using singular value decomposition. BMC Bioinformatics 6. DOI: 10.1186/1471-2105-6-225.

Vento-Tormo R, Efremova M, Botting RA, Turco MY, Vento-Tormo M, Meyer KB, Park JE, Stephenson E, Polański K, Goncalves A, Gardner L, Holmqvist S, Henriksson J, Zou A, Sharkey AM, Millar B, Innes B, Wood L, Wilbrey-Clark A, Payne RP, Ivarsson MA, Lisgo S, Filby A, Rowitch DH, Bulmer JN, Wright GJ, Stubbington MJT, Haniffa M, Moffett A, 
559 Teichmann SA. 2018. Single-cell reconstruction of the early maternal-fetal interface in 560 humans. Nature. DOI: 10.1038/s41586-018-0698-6.

561 Wolf FA, Angerer P, Theis FJ. 2018. SCANPY: Large-scale single-cell gene expression data $562 \quad$ analysis. Genome Biology. DOI: 10.1186/s13059-017-1382-0.

563 Zhang Y, Ma Y, Huang Y, Zhang Y, Jiang Q, Zhou M, Su J. 2020. Benchmarking algorithms for 564

565

566

567

568 
Table $\mathbf{1}$ (on next page)

Functional gene set 
1

\begin{tabular}{lcl}
\hline Function name & Number of gene sets & details \\
\hline C2: Reactome gene sets & 1,499 & $\begin{array}{l}\text { Gene sets derived from the Reactome } \\
\text { pathway database. }\end{array}$ \\
& & $\begin{array}{l}\text { Gene sets that contain genes annotated } \\
\text { by the same GO term. The C5 collection } \\
\text { is divided into three sub-collections } \\
\text { based on GO ontologies: BP, CC, and } \\
\text { MF. }\end{array}$ \\
& 9,996 & $\begin{array}{l}\text { Gene sets that represent cell states and } \\
\text { perturbations within the immune system. } \\
\text { The signatures were generated by } \\
\text { manual curation of published studies in } \\
\text { human and mouse immunology. }\end{array}$ \\
& & \\
C7: immunologic signature gene & 4,872 & \\
sets & &
\end{tabular}

2

3

4 


\section{Table 2 (on next page)}

Top five pathways for mature hepatocyte cells (liver dataset) and beta cells (pancreas dataset) 
1

\begin{tabular}{|c|c|c|}
\hline Function & cluster & $\begin{array}{l}\text { Adjusted p- } \\
\text { value }\end{array}$ \\
\hline reactome-regulation-of-gene-expression-in-beta-cells & beta & $7.47 \mathrm{E}-48$ \\
\hline reactome-regulation-of-beta-cell-development & beta & $1.02 \mathrm{E}-25$ \\
\hline reactome-activation-of-nmda-receptors-and-postsynaptic-events & beta & $1.83 \mathrm{E}-13$ \\
\hline $\begin{array}{l}\text { reactome-negative-regulation-of-tcf-dependent-signaling-by-dvl- } \\
\text { interacting-proteins }\end{array}$ & beta & $2.05 \mathrm{E}-11$ \\
\hline reactome-synthesis-of-pips-at-the-early-endosome-membrane & beta & $1.76 \mathrm{E}-06$ \\
\hline reactome-gluconeogenesis & mature hepatocyte & $1.75 \mathrm{E}-11$ \\
\hline reactome-signaling-by-bmp & mature hepatocyte & $4.50 \mathrm{E}-09$ \\
\hline reactome-apoptotic-cleavage-of-cell-adhesion-proteins & mature hepatocyte & $9.00 \mathrm{E}-09$ \\
\hline $\begin{array}{l}\text { reactome-transport-of-nucleosides-and-free-purine-and-pyrimidine- } \\
\text { bases-across-the-plasma-membrane }\end{array}$ & mature hepatocyte & $1.51 \mathrm{E}-08$ \\
\hline reactome-bbsome-mediated-cargo-targeting-to-cilium & mature hepatocyte & $1.76 \mathrm{E}-08$ \\
\hline
\end{tabular}




\section{Table 3 (on next page)}

Results of the top five GO-based FEM methods for each cluster (PBMC data set) 


\begin{tabular}{|c|c|c|}
\hline Function & cluster & $\begin{array}{l}\text { Adjusted p- } \\
\text { value }\end{array}$ \\
\hline go-mhc-class-ii-receptor-activity & $\mathrm{B}$ & $1.31 \mathrm{E}-299$ \\
\hline go-mhc-class-ii-protein-complex & $\mathrm{B}$ & $1.94 \mathrm{E}-207$ \\
\hline go-clathrin-coated-endocytic-vesicle-membrane & $\mathrm{B}$ & $8.60 \mathrm{E}-183$ \\
\hline go-clathrin-coated-endocytic-vesicle & $\mathrm{B}$ & $1.40 \mathrm{E}-174$ \\
\hline go-mhc-protein-complex-assembly & $\mathrm{B}$ & $2.39 \mathrm{E}-173$ \\
\hline go-collagen-containing-extracellular-matrix & CD14+_Mono & $3.17 \mathrm{E}-235$ \\
\hline go-rage-receptor-binding & CD14+_Mono & $1.42 \mathrm{E}-226$ \\
\hline go-chemokine-production & CD14+- Mono & $1.61 \mathrm{E}-211$ \\
\hline go-defense-response-to-bacterium & CD14+_-Mono & $9.86 \mathrm{E}-194$ \\
\hline go-neutrophil-migration & CD14+_Mono & $2.36 \mathrm{E}-187$ \\
\hline go-cytolysis & $\mathrm{CD} 8 \mathrm{~T}^{-}$ & $2.61 \mathrm{E}-64$ \\
\hline go-t-cell-receptor-complex & CD8_- T & $7.62 \mathrm{E}-53$ \\
\hline go-negative-regulation-by-host-of-viral-transcription & CD8_- T & $5.71 \mathrm{E}-39$ \\
\hline go-regulation-of-cell-cell-adhesion-mediated-by-integrin & CD8_T & $1.50 \mathrm{E}-36$ \\
\hline go-t-cell-receptor-binding & CD8_- T & 4.19E-29 \\
\hline go-ige-binding & $\mathrm{DC}^{-}$ & $4.78 \mathrm{E}-241$ \\
\hline $\begin{array}{l}\text { go-t-cell-activation-via-t-cell-receptor-contact-with-antigen- } \\
\text { bound-to-mhe-molecule-on-antigen-presenting-cell }\end{array}$ & $\mathrm{DC}$ & $6.80 \mathrm{E}-23$ \\
\hline go-mhc-class-ii-receptor-activity & $\mathrm{DC}$ & $1.48 \mathrm{E}-14$ \\
\hline go-hydrolase-activity-acting-on-ester-bonds & $\mathrm{DC}$ & 0.000373 \\
\hline go-lipid-metabolic-process & DC & 0.000529 \\
\hline go-igg-binding & FCGR3A+_Mono & $9.13 \mathrm{E}-143$ \\
\hline go-negative-regulation-of-leukocyte-proliferation & FCGR3A+_Mono & 4.71E-60 \\
\hline go-regulation-of-mast-cell-activation & FCGR3A+_Mono & $7.62 \mathrm{E}-59$ \\
\hline $\begin{array}{l}\text { go-regulation-of-mast-cell-activation-involved-in-immune- } \\
\text { response }\end{array}$ & FCGR3A+_Mono & $1.83 \mathrm{E}-58$ \\
\hline go-dendritic-cell-differentiation & FCGR3A+_Mono & $3.00 \mathrm{E}-58$ \\
\hline go-positive-t-cell-selection & Memory_CD4_T & $5.51 \mathrm{E}-40$ \\
\hline go-positive-thymic-t-cell-selection & Memory_CD4_T & $1.80 \mathrm{E}-37$ \\
\hline go-t-cell-receptor-binding & Memory_CD4_T & $1.89 \mathrm{E}-37$ \\
\hline go-alpha-beta-t-cell-receptor-complex & Memory_CD4_T & $7.89 \mathrm{E}-35$ \\
\hline go-positive-regulation-of-t-cell-receptor-signaling-pathway & Memory_CD4_T & $3.62 \mathrm{E}-33$ \\
\hline go-t-cell-differentiation-in-thymus & Naive_CD4_T & $5.58 \mathrm{E}-123$ \\
\hline go-thymic-t-cell-selection & Naive_CD4_T & 4.23E-87 \\
\hline go-positive-regulation-of-t-cell-receptor-signaling-pathway & Naive_CD4_T & $2.06 \mathrm{E}-58$ \\
\hline go-t-cell-receptor-complex & Naive_CD4_T & $2.18 \mathrm{E}-57$ \\
\hline go-negative-t-cell-selection & Naive_CD4_T & $1.51 \mathrm{E}-39$ \\
\hline go-granzyme-mediated-apoptotic-signaling-pathway & NK & $2.10 \mathrm{E}-190$ \\
\hline go-cytolytic-granule & NK & $1.03 \mathrm{E}-150$ \\
\hline go-positive-regulation-of-natural-killer-cell-chemotaxis & NK & $6.27 \mathrm{E}-128$ \\
\hline go-cytolysis & NK & $6.91 \mathrm{E}-97$ \\
\hline go-ccr5-chemokine-receptor-binding & NK & $9.03 \mathrm{E}-58$ \\
\hline go-platelet-alpha-granule-membrane & Platelet & $6.75 \mathrm{E}-158$ \\
\hline go-platelet-alpha-granule & Platelet & 4.94E-06 \\
\hline go-platelet-degranulation & Platelet & $5.90 \mathrm{E}-06$ \\
\hline go-platelet-alpha-granule-lumen & Platelet & $6.23 \mathrm{E}-06$ \\
\hline go-contractile-fiber & Platelet & $8.58 \mathrm{E}-06$ \\
\hline
\end{tabular}




\section{Figure 1}

Workflow of the proposed method.

(A) Flowchart of the FEM algorithm, which was used to calculate Fisher's exact test for each cell and each gene set, following which the calculated p-value matrix was converted into the FEM through information content. The efficiency of the calculation method was improved by matrix operation and multi-core parallel processing (see the Method section for details) Cluster differential expression analysis based on FEM and integration of FEM and GEM (including $C$ and $D$, the analysis is performed in Seurat). For downstream analysis, FEM can be regarded as another type of GEM. Therefore, Seurat's analysis pipeline can be used to analyse FEM (Figure 1C) or treat FEM and GEM as two different omics data for integrated analysis (Figure 1D). (C) GEM/FEM was used for dimension reduction, clustering, and differential expression genes/function analysis (GC-DEG/FC-DEF). (D) Due to the loss of gene expression information in FEM, the dimensionality reduction and clustering is based on GEM, and differential expression function analysis among clusters in the GEM cluster (GC-DEF) was performed. 


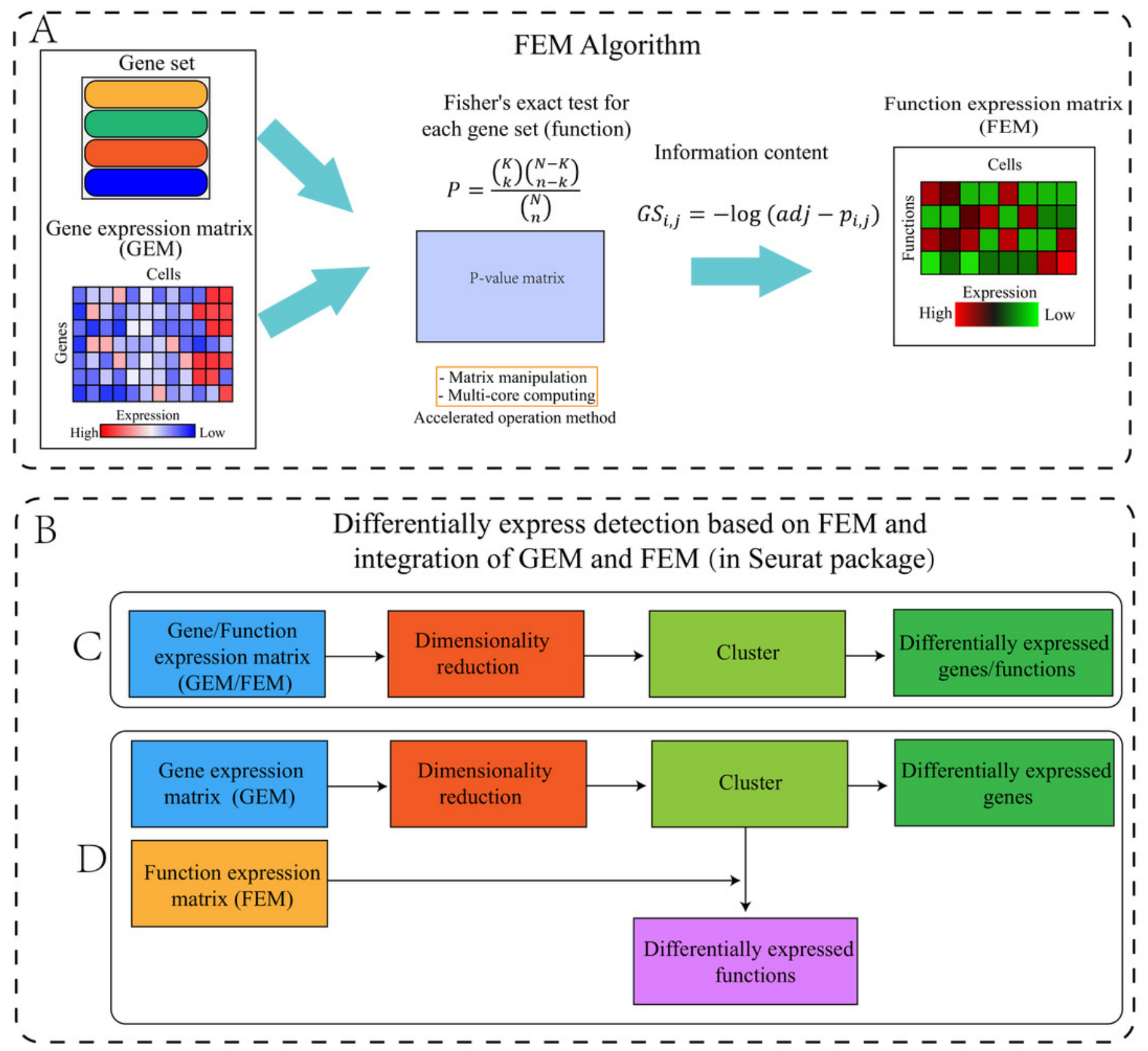




\section{Figure 2}

Algorithm optimization based on matrix multiplication and multi-core operation.

(A) Convert the GEM and the gene sets into a 0,1 matrix. The result of multiplication of the two matrices representing the number of genes expressed in cell <!--[if !msEquation]--> <!-[if !vml]---> <!--[endif]--><!--[endif]--> and set <!--[if !msEquation]--> <!--[if !vml]--><!-[endif]--><!--[endif]-->. (B) The multi-core parallel computing method established two queues. The data queue was used to store the data needed for calculation and the result queue stored the calculated results. Each set of the two queues uniquely identified the cell and the function to which it belonged. The calculation process adopted a multi-process operation. 
A

\section{Matrix manipulation}

Functions
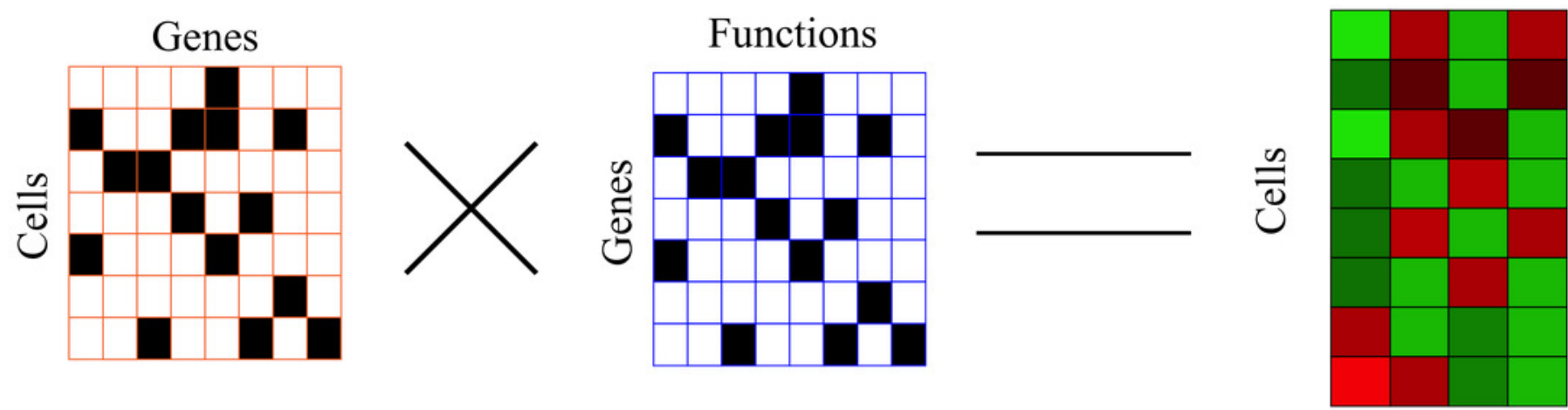

B

Multi-core computing

Data queue

$\begin{array}{lll}\mathrm{N} & \mathrm{K} \\ \mathrm{n} & \mathrm{k}\end{array}$
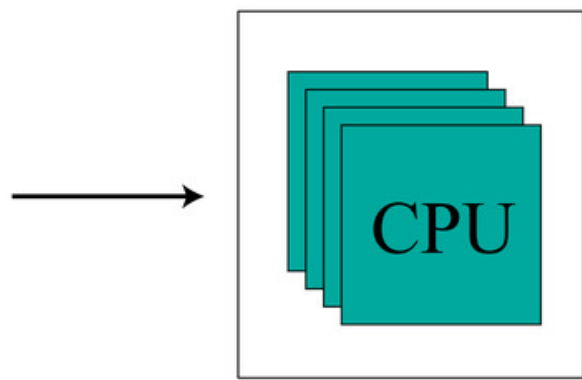

Result queue

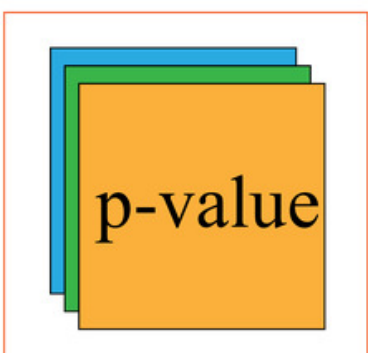




\section{Figure 3}

The comparison between GEM clustering (3A) and GO-based FEM clustering (3B) helps to find the similarities between immune cells.

(A) The results of GEM clustering. (B) The GO-based clustering results. (C) The correspondence between each cluster and cell type of GO-based clustering. (D) The differentiation tree of immune cells (Lim et al., 2013). All clustering results are plotted by UMAP. Figure $\mathrm{C}$ shows that there are three clusters with low SC scores. The G1 cluster is mainly composed of two types of monocytes (CD14+_Mono, FCGR3A+_Mono). It can be seen from Figure 3D that all monocytes have the same recent developmental origin. G2 is composed of NK cells and CD8 T cells. Figure 3D shows that both NK cells and T cells are developed from T/NK cell progenitor cells. G3 is composed of naive CD4 T, CD8 T, and memory CD4 T. The differentiation tree in Figure 3D shows that all T cells have a common nearest ancestor. The No. 7 cluster has a low score, however, it has a small number of cells and the number of cells in all cell types is not the main cluster of the corresponding cell type, and is ignored. 
A

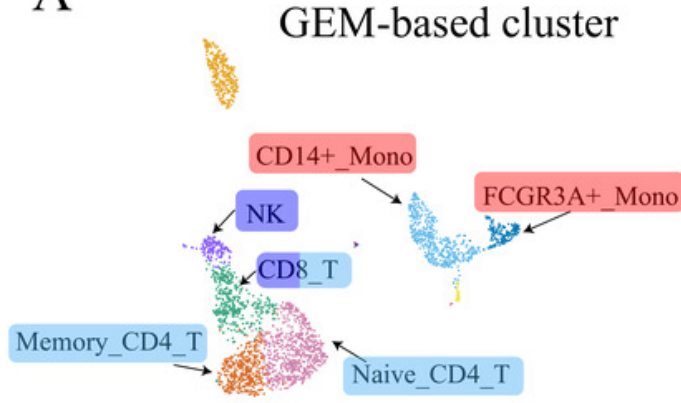

C

\begin{tabular}{|c|c|c|c|c|c|c|c|c|c|}
\hline cell_typelcluster & 4 & 3 & 0 & 2 & 7 & 5 & 1 & 8 & 6 \\
\hline B & 320 & 0 & 2 & 0 & 8 & 0 & 8 & 0 & 12 \\
\hline Naive_CD4_T & 2 & 0 & 418 & 0 & 3 & 0 & 134 & 0 & 127 \\
\hline CD14+_Mono & 0 & 317 & 0 & 175 & 0 & 0 & 0 & 0 & 0 \\
\hline CD8_T & 1 & 0 & 77 & 0 & 3 & 115 & 114 & 0 & 74 \\
\hline NK & 0 & 0 & 0 & 0 & 12 & 135 & 4 & 0 & 3 \\
\hline DC & 0 & 1 & 0 & 29 & 5 & 0 & 0 & 0 & 0 \\
\hline FCGR3A+_Mono & 0 & 18 & 0 & 143 & 0 & 0 & 0 & 0 & 0 \\
\hline Platelet & 0 & 0 & 0 & 1 & 0 & 0 & 1 & 11 & 0 \\
\hline Memory_CD4_T & 1 & 0 & 116 & 0 & 12 & 3 & 289 & 0 & 6 \\
\hline SC & 0.99 & \begin{tabular}{|l|} 
\\
\end{tabular} & 0.68 & 0.5 & 0.28 & 0.53 & 0.53 & 1 & 0.57 \\
\hline
\end{tabular}

$B$
B

FEM-based cluster

CD14+ Mono

CD8_T

DC

- FCGR3A+ Mono

- Memory CD4 T

- Naive_CD4_T

- NK

Platelet

FCGR3A+_Mono

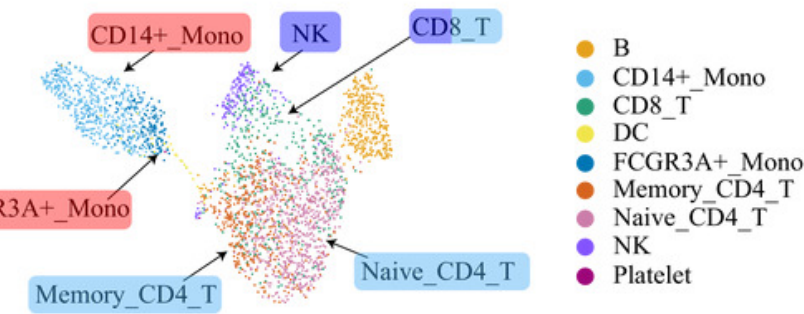

D

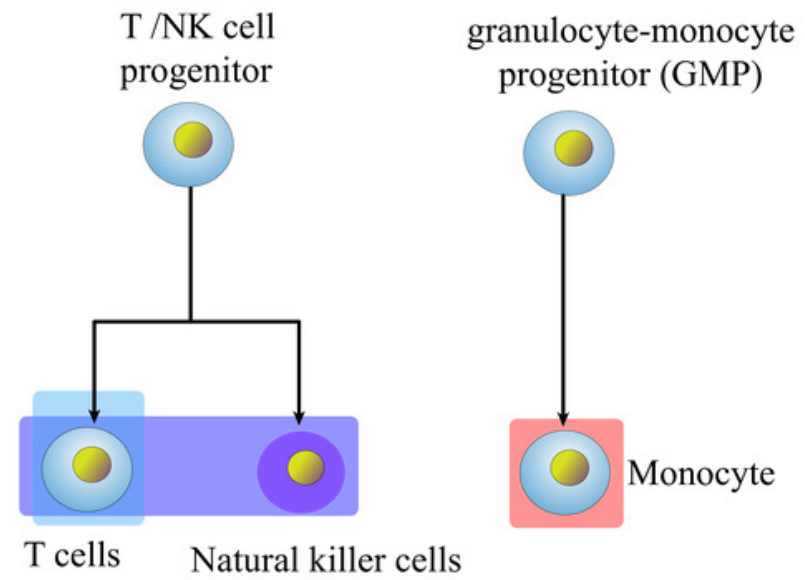




\section{Figure 4}

Comparison of GEM clustering (4A) and GO-based FEM clustering (4B) helps to find the similarities between liver cells.

(A) The results of GEM clustering. (B) The GO-based clustering results. (C) The correspondence between each cluster and cell type of GO-based clustering. (D) The developmental trajectory of stem cells (Camp et al., 2017). All clustering results are plotted by UMAP. Figure $4 C$ shows that a cell group has a low SC score. G1 is mainly composed of definitive endoderm and hepatic endoderm cells. These two types of cells are far apart in GEM (Figure 4A). But it is close to each other in GO-based FEM cluster. From Figure 4D, we find that these two cells are adjacent to each other on the cell differentiation trajectory, and they are the progenitor cells of other cells in the liver. 
A

\section{GEM-based cluster}

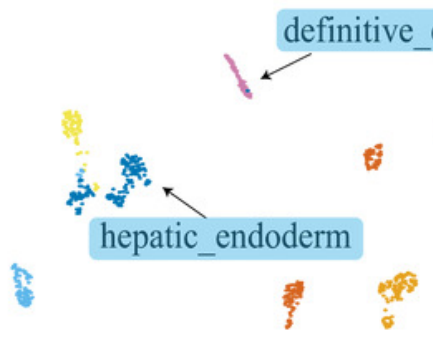

C

\begin{tabular}{|l|c|c|c|c|c|c|c|c|c|c|}
\hline cell_typelcluster & 7 & 5 & 4 & 1 & 3 & 8 & 9 & 0 & 6 & 2 \\
\hline ipsc & 0 & 0 & 0 & 0 & 0 & 0 & 0 & 0 & 0 & 80 \\
\hline endothelial & 1 & 0 & 73 & 1 & 0 & 52 & 0 & 0 & 0 & 0 \\
\hline immature_hepatoblast & 0 & 2 & 0 & 0 & 2 & 0 & 0 & 10 & 67 & 0 \\
\hline mature_hepatocyte & 0 & 0 & 0 & 0 & 70 & 0 & 0 & 8 & 3 & 0 \\
\hline definitive_endoderm & 3 & 36 & 0 & 0 & 0 & 0 & 0 & 31 & 0 & 0 \\
\hline mesenchymal_stem_cell & 63 & 0 & 1 & 91 & 0 & 0 & 16 & 0 & 0 & 0 \\
\hline hepatic_endoderm & 0 & 34 & 0 & 0 & 3 & 2 & 0 & 127 & 1 & 0 \\
\hline sc & 0.94 & 0.5 & 0.99 & 0.99 & 0.93 & 0.96 & 1 & 0.72 & 0.94 & 1 \\
\hline
\end{tabular}

B

\section{FEM-based cluster}

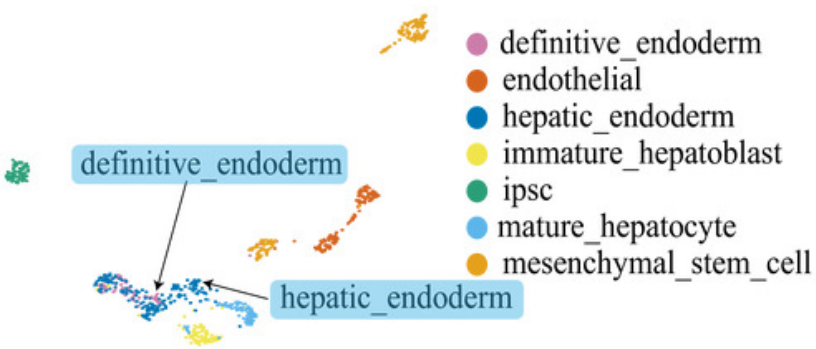

D

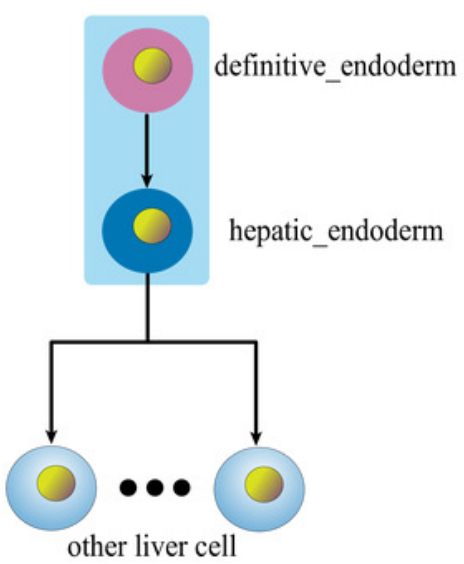




\section{Figure 5}

Comparison of GEM clustering (5A) and GO-based FEM clustering (5B) helps to find the similarities between pancreatic cells.

(A) The results of GEM clustering. (B) The GO-based clustering results. (C) The correspondence between each cluster and cell type of GO-based clustering and the SC score.

(D) The differentiation lineage of pancreatic cells (Jacobson \& Tzanakakis, 2017) . All clustering results are plotted by UMAP. Figure $5 \mathrm{C}$ shows that the two cell groups have the lowest scores. Although G1 has a low score, it has a small number of cells. The number of cells in all cell types is not the main cluster of the corresponding cell type, so it will be ignored. G2 is composed of five types of cells (alpha, beta, delta, gamma, epsilon). Although the epsilon cell has only two cells in cluster 1, but it has only three cells, so it is considered that it is distributed in G2. These five types of cells are far apart in the GEM UMAP plot, but close to each other in the GO-based FEM plot. Figure 5D shows that all these cells have a common progenitor cell differentiation. 


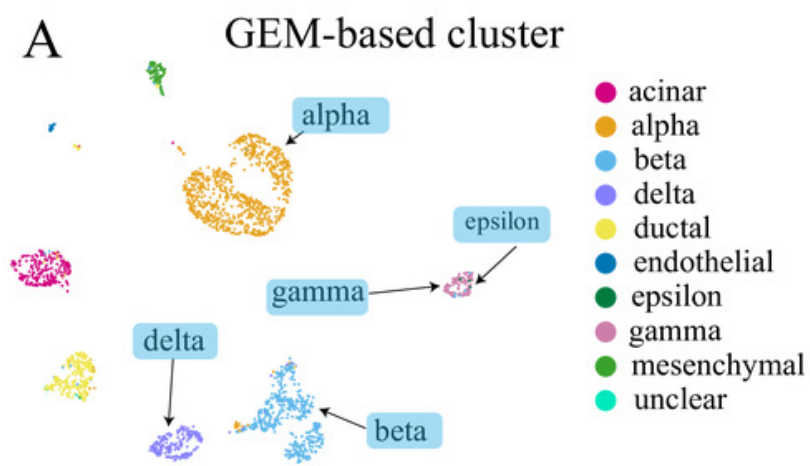

C

\begin{tabular}{|l|c|c|c|c|c|c|c|c|c|c|}
\hline cell_typelcluster & 6 & 9 & 8 & 3 & 7 & 0 & 4 & 2 & 5 & 1 \\
\hline alpha & 5 & 0 & 8 & 303 & 0 & 13 & 5 & 318 & 2 & 158 \\
\hline endothelial & 1 & 0 & 0 & 0 & 20 & 0 & 0 & 0 & 0 & 0 \\
\hline acinar & 3 & 0 & 0 & 0 & 0 & 0 & 10 & 0 & 204 & 2 \\
\hline mesenchymal & 79 & 0 & 0 & 0 & 0 & 0 & 1 & 0 & 0 & 0 \\
\hline delta & 0 & 0 & 1 & 6 & 0 & 79 & 0 & 7 & 0 & 100 \\
\hline ductal & 2 & 14 & 0 & 0 & 0 & 3 & 216 & 0 & 9 & 1 \\
\hline unclear & 0 & 0 & 0 & 0 & 0 & 0 & 4 & 0 & 0 & 0 \\
\hline epsilon & 0 & 0 & 0 & 1 & 0 & 0 & 0 & 0 & 0 & 2 \\
\hline beta & 2 & 1 & 9 & 4 & 0 & 368 & 7 & 6 & 1 & 50 \\
\hline gamma & 0 & 0 & 0 & 16 & 0 & 8 & 0 & 2 & 2 & 73 \\
\hline sc & 0.86 & 0.93 & 0.5 & 0.92 & 1 & 0.78 & 0.89 & 0.95 & 0.94 & 0.41 \\
\hline
\end{tabular}

B FEM-based cluster

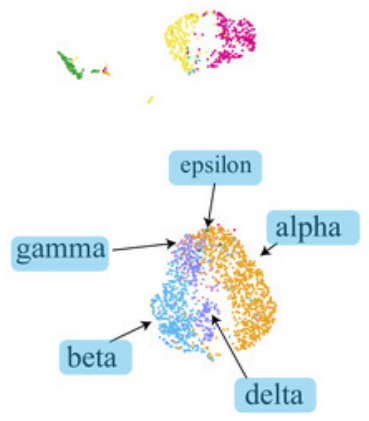

acinar

- beta

- delta

ductal

endothelial

- epsilon

gamma

- mesenchymal

unclear

D

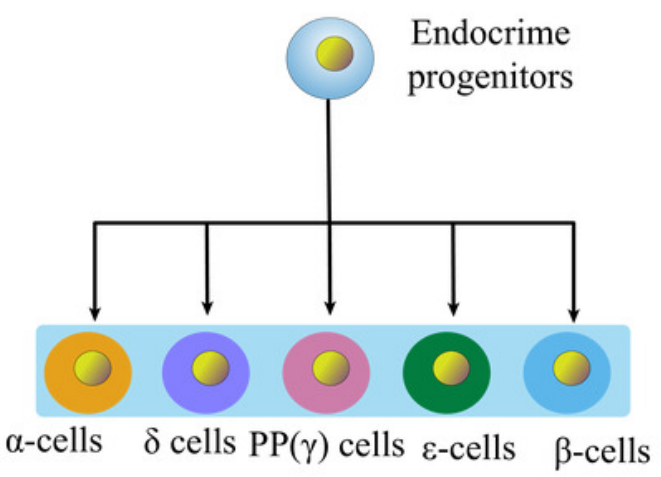




\section{Figure 6}

An example of GC-DEF functional analysis.

(A) Official clustering results. (B) Results of GEM without cell filtering show a small cell group above the NK cell cluster (when compared with Figure 5A). (C) By directly displaying the expression value of specific pathways in all cells, this cell group was found to have high expression of the cell proliferation-related pathway. For downstream analysis, cell screening may result in some cells being filtered out, which may hold some interest. In the process of biological research, cells at special stages often account for only a small part of the total number of cells, but they may be important for explaining biological processes. For example, a small part of cells in the cell cycle stage (Figure B). They are clustered in the NK cell group in clustering, but compared with other NK cells, they are a prominent part of the UMAP plot. When doing differential expression between cell groups, the signals of these proliferating cells will be masked by NK cells. The FEM method is based on a single cell, so it can directly display the functions of these special cells even at single cell level. 


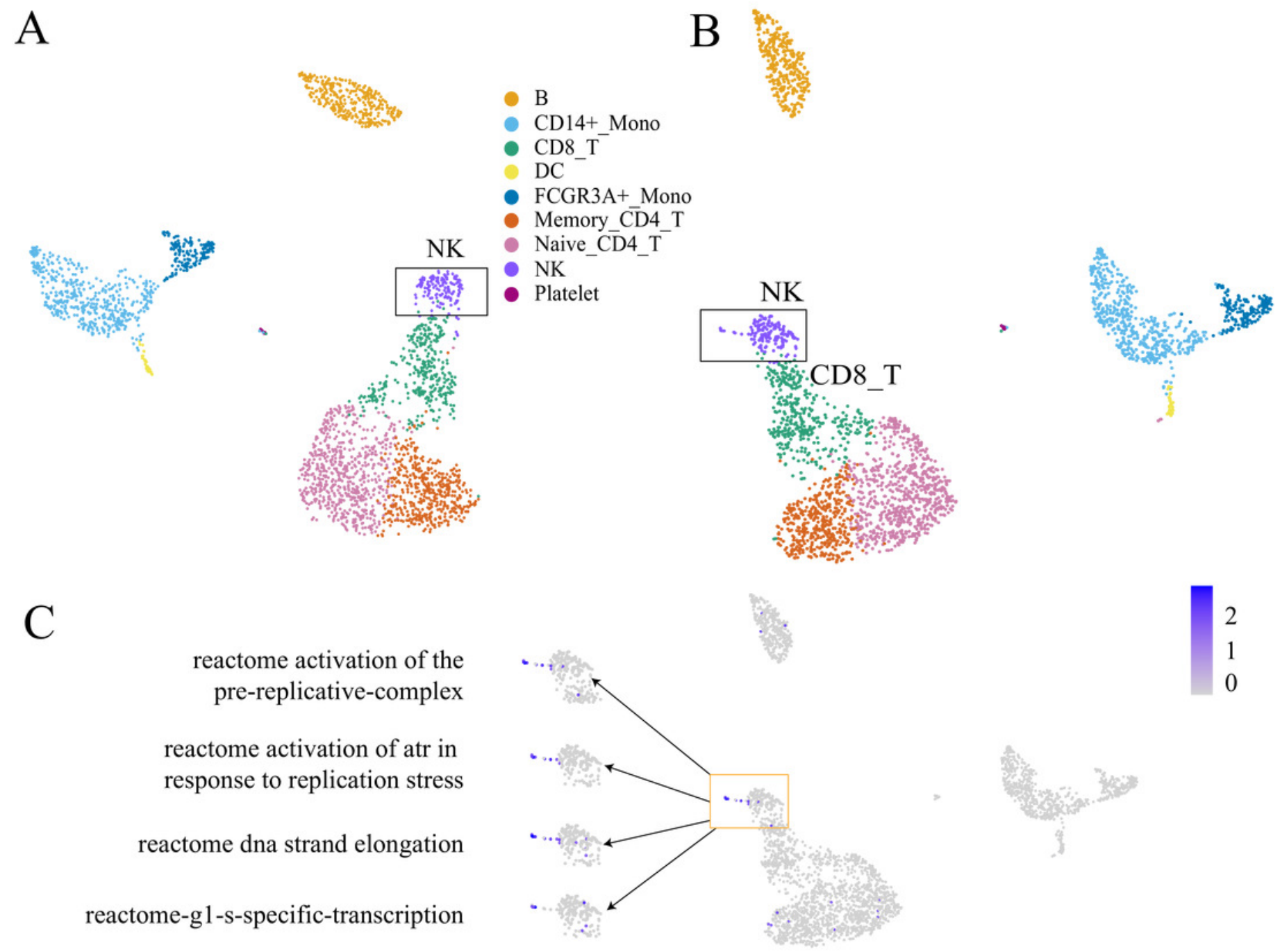




\section{Figure 7}

Validation results of Immunologic Signatures Collection gene sets.

ImmuneSigDB (Godec et al., 2016) is a collection of gene sets of human and mouse immune cells collected from the GEO database. All data are manually corrected. Each gene set represents genes that are highly expressed in a specific immune cell type compared to another or all other cell types. Here, we verify whether the expression of each gene set (in this case, functional expression) is consistent with the corresponding cell type. (A) UMAP plot for each cell type based on GEM. (B-G) The expression values of various cell type-specific gene sets in each cell. The figures show that the functional expression obtained by our method is consistent with the corresponding cell type.

A

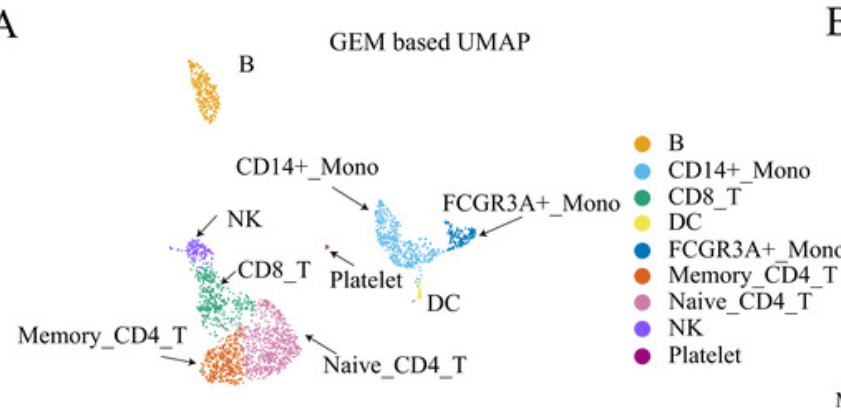

B gse26495-naive-vs-pd1low-cd8-tcell-up C

gse22886 naive tcell vs monocyte up

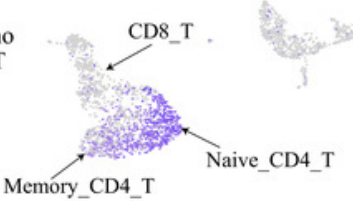

$\mathrm{F}$ gse11057 pbmc vs mem cd4 tcell up
D gse7764 nkcell vs splenocyte up

E gse10325 myeloid tcell vs cd4 up
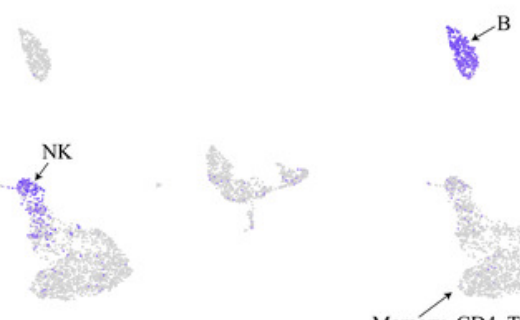

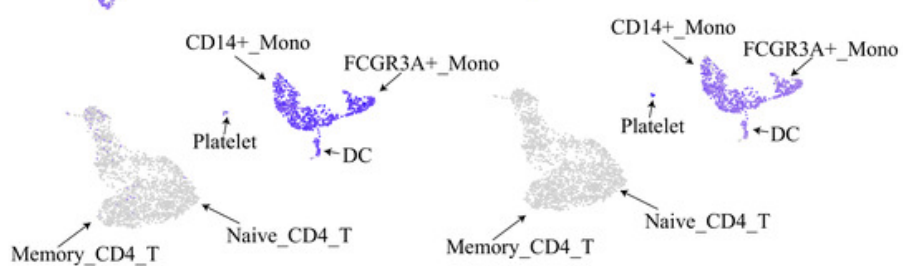

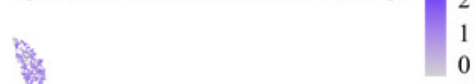

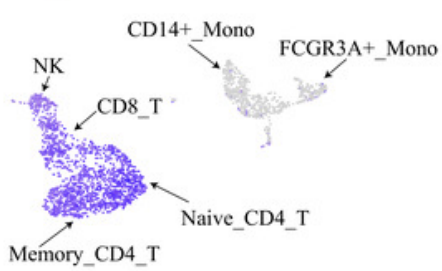

$\mathrm{G}$ gse22886 naive bcell vs monocyte up 衰- ${ }^{-\mathrm{B}}$

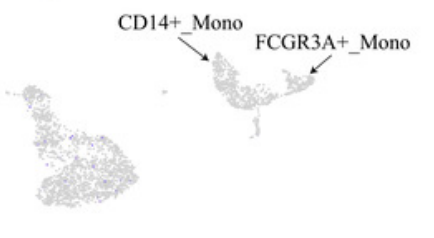

